\title{
ABSORPTION STUDIES OF THE SULPHUR DONOR IN GaSb*
}

\author{
P. KACZOR ${ }^{a}$, A.M. Gerrits ${ }^{b}$, L. DobACZEWSKI ${ }^{a}$, Z. KALIŃSKI ${ }^{a}$, \\ AND A. WitTLIN ${ }^{b}$ \\ ${ }^{a}$ Institute of Physics, Polish Academy of Sciences \\ Al. Lotników 32/46, 02-668 Warszawa, Poland \\ ${ }^{b}$ High Field Magnet Laboratory - University of Nijmegen \\ P.O. Box 9010, 6500 GL Nijmegen, The Netherlands
}

The shallow-deep bistability of the sulphur-related DX centre in GaSb is demonstrated. After photoexcitation of the defect with the near-band gap light the metastable inter-donor transition in the far infrared can be observed. This observation allowed us to evaluate the polaron effective mass and polaron coupling constant for the material.

PACS numbers: 71.55.Eq, 71.70.Ej, 78.50.Ge

Although the DX centres in III-V semiconductors belong to the most investigated defect systems in last fifteen years there are still some unsolved problems. Among them there is a microscopic identification of processes leading to metastability effects observed for the sulphur donor in $\mathrm{GaSb}$. This donor forms in $\mathrm{GaSb}$ the DX state possible to observe at ambient pressure in a binary compound. Its unusual features were known since early seventies [1].

In this study results of the metastable infrared absorption measurements in $\mathrm{GaSb}$ are presented. We show, for the first time, the inter-donor absorption of a shallow donor in this material. The existence of the metastable IR-absorption band related to a single shallow donor is a characteristic feature of the shallow-deep bistability common to many DX-type defects in, e.g., $\mathrm{CdF}_{2}$ [2] and AlGaAs [3]. Present results give a strong argument in favour of the "single impurity" character of the deep sulphur donor in GaSb.

The samples used in our experiments were cut from Czochralski grown $\mathrm{GaSb}$ crystals doped with sulphur. During the growth the undoped $p$-type material was overcompensated which resulted in $n$-type of conductivity with the net free electron concentration around $10^{16} \mathrm{~cm}^{-3}$. The Hall measurements performed in the temperature range $40-300 \mathrm{~K}$ showed the persistent photoconductivity effect (PPC) [4].

*This work has been financially supported by the grant No. 2 P302 11705 of the State Committee for Scientific Research (Republic of Poland). 
The absorption measurements were performed on the Bruker ATS 113 spectrometer coupled to the $18 \mathrm{~T}$ superconducting magnet. Two beam splitters were used to cover the range from 15 to $700 \mathrm{~cm}^{-1}$. This was sufficient to observe the Zeeman splitting with magnetic fields ranging from 0 to 15 tesla. The measurement temperature was $1.7 \mathrm{~K}$.

The sample was cooled down in dark, i.e., only far infrared light (with $\nu$ below approximately $1500 \mathrm{~cm}^{-1}$ ) could reach the sample through the black polyethylene filters. Although a mercury lamp was used as a source and the measurements were performed usually longer than 8 hours this incident light caused no observable PPC effect. Only when the sample was illuminated with strong near infrared radiation the metastable absorption band appeared peaking at approximately $68 \mathrm{~cm}^{-1}$. Its intensity scaled with illumination time till it reached saturation. It did not change in any other way during the measurement (over 8 hours). This band splitted in the magnetic field into two components. One of them, for lower fields, shifted slightly to the low energy side of the spectrum and remained unchanged at $54-56 \mathrm{~cm}^{-1}$ for higher fields. The other one moved quickly with increasing field towards higher energies (Fig. 1). The latter peak (especially at higher fields) increased while the

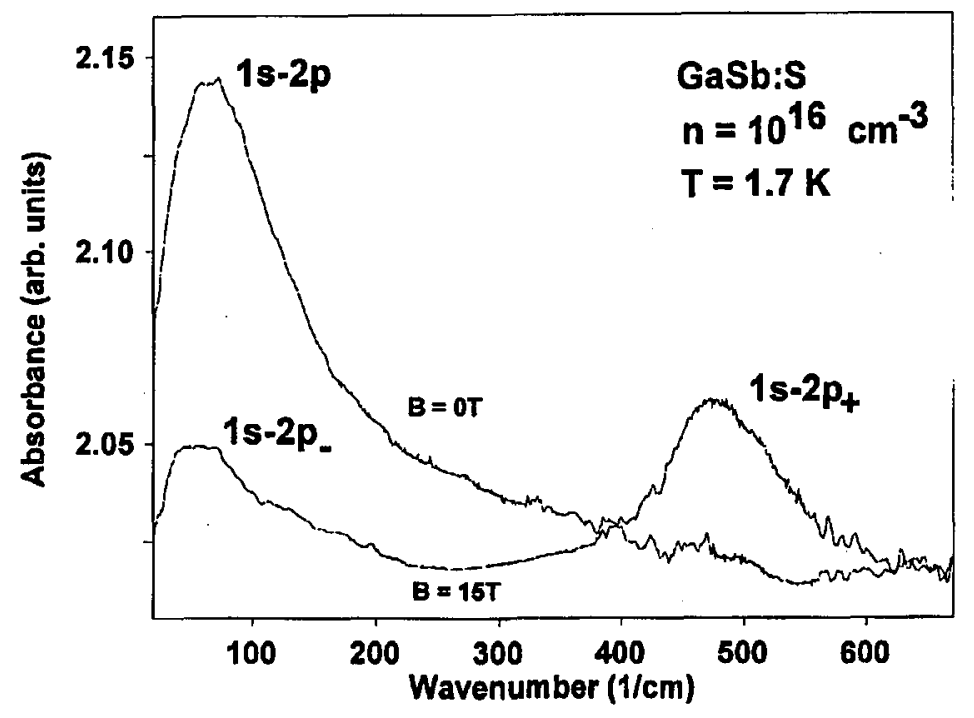

Fig. 1. Zeeman splitting of the metastable far infrared inter-donor transition.

first one decreased with the magnetic field. Such a behaviour with the field is very characteristic of the $1 s-2 p$ inter-donor transition of the shallow hydrogenic donor which is subjected to the Zeeman splitting. One can then attribute the low and high energy peaks to the $1 s-2 p_{-}$and $1 s-2 p_{+}$transitions, respectively.

The striking feature of the $1 s-2 p_{+}$transition is a pronounced discontinuity in the peak position vs. field dependence in the vicinity of the $\mathrm{LO}$ and TO phonon absorption (i.e. around $200 \mathrm{~cm}^{-1}$ ). The positions of this peak in the low magnetic field branch deviate systematically downwards from straight line and at the higher 
fields upwards. This downward and upward deviation and the resulting discontinuity indicate that there is the electron-phonon coupling of the $2 p_{+}$state to the LO phonon.

From the magnetic field dependence of the $\Delta E=E\left(1 s-2 p_{+}\right)-E\left(1 s-2 p_{-}\right)$ splitting one can estimate the effective mass at the conduction band minimum according to the formula: $\Delta E=h \omega_{\mathrm{c}} / 2 \pi$, where $\omega_{\mathrm{c}}=e B / m_{\mathrm{eff}}$ is the cyclotron resonance frequency, $B$ - magnetic field, and $m_{\mathrm{eff}}-$ effective mass. The effective mass obtained in this way: $m_{\mathrm{eft}}=0.0368 m_{0}$ is not a pure electronic one as the polaron coupling is present. However, its order of magnitude indicates that the wave functions of the electron bound to the donor originate from the $\Gamma$ minimum of the conduction band.

The non-polaron and polaron effective masses are related via the formula (for weak coupling): $m_{\mathrm{eff}}^{\mathrm{p}}=(1-\alpha / 6)^{-1} m_{\mathrm{eff}}^{\mathrm{np}}$, where $\alpha$ is the polaron coupling constant. The value of $\alpha$ can be deduced if the value of the discontinuity around the Restrahlen frequency is known : $\Delta \nu_{\text {disc }}=\alpha \nu_{\mathrm{LO}}$ ( $\nu_{\mathrm{LO}}$ is the LO-phonon frequency). From our results we get the value of $\Delta \nu_{\text {disc }}$ (with no theoretical fit) approximately equal to $130 \mathrm{~cm}^{-1}$, and therefore, $\alpha=0.58$ (for $\nu_{\mathrm{LO}}=232.6 \mathrm{~cm}^{-1}$ ), and the non-polaron effective mass $m_{\mathrm{eff}}^{\mathrm{np}}=0.0332 m_{0}$.

In comparison to the cyclotron resonance data for $\mathrm{GaSb}$ [5], the value of $\alpha$ and $m_{\mathrm{eff}}{ }^{\mathrm{np}}$ obtained in Ref. [5] $\left(m_{\mathrm{eff}}^{\mathrm{np}}=0.0396 \mathrm{~m}_{0}\right)$ does not seem to differ much from ours when one takes into account the error made by estimating $h \omega_{\mathrm{c}} / 2 \pi$ from the above formulae. The main source of error in $h \omega_{c} / 2 \pi$ evaluation was the estimation of the peak position for the $1 s-2 p_{-}$transition. This could not be done with a sufficient certainty as the spectra are truncated at low energies. Furthermore, the parameter $\alpha$ should be evaluated by a fitting of the experimental points to the exact theoretical model (e.g. given in Ref. [6]). The value of $\alpha=0.025$ used in Ref. [5] was calculated for "hot electrons", a system completely different from the one investigated, therefore the difference in $\alpha$ should not surprise too much. We would rather expect $\alpha$ to be in order of $0.3-0.5$ as in Ref. [6]. Finally, the energy of the $1 s-2 p$ transition would give the donor binding energy equal to $11 \mathrm{meV}$. This is much too much in comparison to the value which could be derived from the effective mass theory (approximately $2 \mathrm{meV}$ ). This indicates a substantial chemical shift for the donor.

In conclusion, it has been experimentally shown that the sulphur-related DX centre in GaSb exhibits the shallow-deep bistability phenomenon. After the excitation with the near-band gap light the metastable inter-donor transition in the far infrared appears. Until now, this observation is only preliminarily correlated with the Hall measurements and absorption measurements near the band gap edge (similarly to Ref. [7]). Further experiments should allow to evidence the one to one correspondence between the deep and shallow metastable states of the sulphur defect in $\mathrm{GaSb}$.

\section{References}

[1] A.I. Vul, A.I. Shik, Fiz. Tekh. Poluprovodn. 8, 1952 (1974).

[2] U. Piekara, J.M. Langer, B. Krukowska-Fulde, Solid State Commun. 23, 583 (1977). 
[3] J.E. Dmochowski, L. Dobaczewski, J.M. Langer, W. Jantsch, Phys. Rev. B 40, 9671 (1989).

[4] U. Wilke, P. Kaczor, unpublished.

[5] D.A. Hill, C.F. Schwerdtfeger, J. Phys. Chem. Solids 33, 1533 (1974).

[6] D.R. Cohn, D.M. Larsen, B. Lax, Phys. Rev. B 6, 1367 (1972).

[7] P. Kaczor, Z.R. Żytkiewicz, M. Godlewski, L. Dobaczewki, Phys. Rev. B 47, 12558 (1993). 